\title{
Bootstrap Statistical Inference for the Variance Based on Fuzzy Data
}

\author{
Mohammad Ghasem Akbari ${ }^{1}$ and Abdolhamid Rezaei ${ }^{2}$ \\ ${ }^{1}$ University of Birjand, Southern Khorasan, Iran \\ ${ }^{2}$ Ferdowsi University of Mashdad, Mashdad, Iran
}

\begin{abstract}
The bootstrap is a simple and straightforward method for calculating approximated biases, standard deviations, confidence intervals, testing statistical hypotheses, and so forth, in almost any nonparametric estimation problem. In this paper we describe a bootstrap method for variance that is designed directly for hypothesis testing in case of fuzzy data based on Yao-Wu signed distance.
\end{abstract}

Zusammenfassung: Der Bootstrap ist eine einfache und geradlinige Methode um in fast jedem nichtparametrischen Schätzproblem geschätzte Biases, Standardabweichungen, Konfidenzintervalle zu berechnen, wie auch statistische Hypothesen zu testen und so weiter. In diesem Aufsatz beschreiben wir eine Bootstrapmethode für die Varianz, welche unmittelbar für Hypothesentests im Falle von unscharfen Daten basierend auf Yao-Wu vorzeichenbehafteter Distanzen ausgelegt ist.

Keywords: Fuzzy Canonical Number, Yao-Wu Signed Distance, Confidence Interval, Testing Hypotheses, Degree of Acceptance, Degree of Rejection.

\section{Introduction}

Statistical analysis in traditional form is based on crispness of data, random variables, point estimations, hypotheses, and so on. There are many different situations in which such concepts are imprecise. On the other hand, the theory of fuzzy sets is a well known tool for the formulation and the analysis of imprecise and subjective concepts. Therefore, confidence intervals and testing hypotheses with fuzzy data can be important. Methods for statistical inference (confidence intervals and hypothesis tests) in fuzzy environments are developed in different approaches.

Filzmoser and Viertl (2004) present a test based on fuzzy values by introducing the fuzzy $p$-value. Torabi, Behboodian, and Taheri (2006) try to develop a new approach for testing fuzzy hypotheses when the available data are fuzzy, too. They state and prove a generalized Neyman-Pearson Lemma for such problems. Some methods of statistical inference with fuzzy data are reviewed by Viertl (2006). Buckley $(2005,2006)$ studies the problems of statistical inference in the fuzzy environment. Thompson and Geyer (2007) proposed the fuzzy $p$-value in latent variable problems. Taheri and Arefi (2008) exhibit an approach to test fuzzy hypotheses based on fuzzy test statistics.

The bootstrap using fuzzy data is developed in different approaches. Montenegro, Colubi, Casals, and Gil (2004) present asymptotic one-sample procedures. The asymptotic development of Körner (2000) concerns general fuzzy random variables (taking values in the space of compact convex fuzzy sets of a finite-dimensional Euclidean space). In 
Gonzalez-Rodriguez, Montenegro, Colubi, and Gil (2006) it is shown that the one-sample method of testing the mean of a fuzzy random variable can be extended to general ones (more precisely, to those whose range is not necessarily finite and whose values are fuzzy subsets of a finite-dimensional Euclidean space).

In this paper we construct a new method for bootstrap testing hypotheses in a fuzzy environment which is completely different from those mentioned before. For this purpose we organize the matter in the following way: In Section 2 we describe some basic concepts of canonical fuzzy numbers and the Yao and Wu (2000) signed distance. In Section 3 we come up with crisp and fuzzy bootstrap confidence intervals for the variance. In Section 4 we summarize the testing of crisp and fuzzy hypotheses.

\section{Preliminaries}

In this section we study canonical fuzzy numbers and the Yao-Wu singed distance.

\subsection{Canonical Fuzzy Numbers}

Let $X$ be the universal space, then a fuzzy subset $\widetilde{x}$ of $X$ is defined by its membership function $\mu_{\widetilde{x}}: X \rightarrow[0,1]$. We denote by $\widetilde{x}_{\alpha}=\left\{x: \mu_{\widetilde{x}}(x) \geq \alpha\right\}$ the $\alpha$-cut set of $\widetilde{x}$ and $\widetilde{x}_{0}$ is the closure of the set $\left\{x: \mu_{\widetilde{x}}(x)>0\right\}$, and

(1) $\widetilde{x}$ is called a normal fuzzy set, if there exists a $x \in X$ such that $\mu_{\widetilde{x}}(x)=1$,

(2) $\widetilde{x}$ is called a convex fuzzy set, if $\mu_{\widetilde{x}}(\lambda x+(1-\lambda) y) \geq \min \left(\mu_{\widetilde{x}}(x), \mu_{\widetilde{x}}(y)\right)$ for all $\lambda \in[0,1]$,

(3) the fuzzy set $\widetilde{x}$ is called a fuzzy number, if $\widetilde{x}$ is a normal convex fuzzy set and its $\alpha$-cut sets are bounded $\forall \alpha \neq 0$,

(4) $\widetilde{x}$ is called a closed fuzzy number, if $\widetilde{x}$ is a fuzzy number and its membership function $\mu_{\widetilde{x}}$ is upper semicontinuous,

(5) $\widetilde{x}$ is called a bounded fuzzy number, if $\widetilde{x}$ is a fuzzy number and its membership function $\mu_{\widetilde{x}}$ has compact support.

If $\widetilde{x}$ is a closed and bounded fuzzy number with $x_{\alpha}^{L}=\inf \left\{x: x \in \widetilde{x}_{\alpha}\right\}$ and $x_{\alpha}^{U}=$ $\sup \left\{x: x \in \widetilde{x}_{\alpha}\right\}$ and its membership function is strictly increasing on the interval $\left[x_{\alpha}^{L}, x_{1}^{L}\right]$ and strictly decreasing on the interval $\left[x_{1}^{U}, x_{\alpha}^{U}\right]$ for any $\alpha \in[0,1]$, then $\widetilde{x}$ is called canonical fuzzy number.

Let “ $\odot$ " be a binary operation $\oplus$ or $\ominus$ between two canonical fuzzy numbers $\widetilde{a}$ and $\widetilde{b}$. The membership function of $\widetilde{a} \odot \widetilde{b}$ is defined by

$$
\mu_{\widetilde{a} \odot \widetilde{b}}(z)=\sup _{x \circ y=z} \min \left\{\mu_{\widetilde{a}}(x), \mu_{\widetilde{b}}(y)\right\}, \quad \forall z \in \mathbb{R},
$$

for $\odot \in\{\oplus, \ominus\}$ and $\circ \in\{+,-\}$.

In the following let $\odot_{\text {int }}$ denote a binary operation $\oplus_{\text {int }}$ or $\ominus_{\text {int }}$ between two closed intervals $\widetilde{a}_{\alpha}=\left[a_{\alpha}^{L}, a_{\alpha}^{U}\right]$ and $\widetilde{b}_{\alpha}=\left[b_{\alpha}^{L}, b_{\alpha}^{U}\right]$. Then $\widetilde{a}_{\alpha} \odot_{i n t} \widetilde{b}_{\alpha}$ is defined as

$$
\widetilde{a}_{\alpha} \odot_{i n t} \widetilde{b}_{\alpha}=\left\{z \in \mathbb{R}: z=x \circ y, x \in \widetilde{a}_{\alpha}, y \in \widetilde{b}_{\alpha}\right\} .
$$


If $\widetilde{a}$ and $\widetilde{b}$ are two closed fuzzy numbers, then $\widetilde{a} \oplus \widetilde{b}$ and $\widetilde{a} \ominus \widetilde{b}$ are also closed fuzzy numbers. Furthermore, we have

$$
\begin{aligned}
& (\widetilde{a} \oplus \widetilde{b})_{\alpha}=\widetilde{a}_{\alpha} \oplus_{i n t} \widetilde{b}_{\alpha}=\left[a_{\alpha}^{L}+b_{\alpha}^{L}, a_{\alpha}^{U}+b_{\alpha}^{U}\right], \\
& (\widetilde{a} \ominus \widetilde{b})_{\alpha}=\widetilde{a}_{\alpha} \ominus_{i n t} \widetilde{b}_{\alpha}=\left[a_{\alpha}^{L}-b_{\alpha}^{U}, a_{\alpha}^{U}-b_{\alpha}^{L}\right] .
\end{aligned}
$$

\subsection{Yao-Wu Signed Distance}

Now we define a signed distance between fuzzy numbers which is used later. Several ranking methods have been proposed so far by Cheng (1998), Modarres and Sadi-Nezhad (2001), and Nojavan and Ghazanfari (2006). In this paper we use another ranking system for canonical fuzzy numbers, which is very realistic and is defined by Yao and Wu (2000) as the following:

Definition 1: For each $a, b \in \mathbb{R}$ define the signed distance $d^{*}$ of $a$ and $b$ by $d^{*}(a, b)=a-b$. Thus, we have the following way to define the rank of any two numbers on $\mathbb{R}$. For each $a, b \in \mathbb{R}$

$$
\begin{aligned}
& d^{*}(a, b)>0 \quad \Leftrightarrow \quad d^{*}(a, 0)>d^{*}(b, 0) \quad \Leftrightarrow \quad a>b, \\
& d^{*}(a, b)<0 \quad \Leftrightarrow \quad d^{*}(a, 0)<d^{*}(b, 0) \quad \Leftrightarrow \quad a<b, \\
& d^{*}(a, b)=0 \quad \Leftrightarrow \quad d^{*}(a, 0)=d^{*}(b, 0) \quad \Leftrightarrow \quad a=b .
\end{aligned}
$$

Definition 2: For each $\widetilde{a}, \widetilde{b}$ (arbitrary canonical fuzzy numbers), define the signed distance of $\widetilde{a}$ and $\widetilde{b}$ as

$$
d(\widetilde{a}, \widetilde{b})=\int_{0}^{1}\left(M_{\alpha}(\widetilde{a})-M_{\alpha}(\widetilde{b})\right) d \alpha=\int_{0}^{1} d^{*}\left(M_{\alpha}(\widetilde{a}), M_{\alpha}(\widetilde{b})\right) d \alpha
$$

where $M_{\alpha}(\widetilde{a})$ and $M_{\alpha}(\widetilde{b})$ equal $\left(a_{\alpha}^{L}+a_{\alpha}^{U}\right) / 2$ and $\left(b_{\alpha}^{L}+b_{\alpha}^{U}\right) / 2$, respectively. Furthermore, $d(\widetilde{a}, \widetilde{b})$ means the distance of $\widetilde{a}$ to $\widetilde{b}$.

Definition 3: (Yao and Wu, 2000) For each $\widetilde{a}, \widetilde{b}$ (arbitrary canonical fuzzy numbers) define the rankings $\prec, \succ$, and $\approx$ of $\widetilde{a}$ and $\widetilde{b}$ by

$$
\begin{aligned}
& d(\widetilde{a}, \widetilde{b})>0 \quad \Leftrightarrow \quad(\widetilde{a}, 0)>d(\widetilde{b}, 0) \quad \Leftrightarrow \quad \widetilde{a} \succ \widetilde{b}, \\
& d(\widetilde{a}, \widetilde{b})<0 \quad \Leftrightarrow \quad d(\widetilde{a}, 0)<d(\widetilde{b}, 0) \quad \Leftrightarrow \quad \widetilde{a} \prec \widetilde{b}, \\
& d(\widetilde{a}, \widetilde{b})=0 \quad \Leftrightarrow \quad d(\widetilde{a}, 0)=d(\widetilde{b}, 0) \quad \Leftrightarrow \quad \widetilde{a} \approx \widetilde{b} .
\end{aligned}
$$

\section{Bootstrap Confidence Interval for Variances}

In this section we introduce a way to get bootstrap crisp and fuzzy confidence intervals based on fuzzy data. Through the use of the bootstrap based on fuzzy observations we obtain accurate intervals without having to make use of the normal theory. This procedure estimates the $\chi^{2}$-distribution directly from the fuzzy data. Here is the bootstrap method in more detail. 


\subsection{Crisp Confidence Interval}

Suppose that we have a canonical fuzzy random sample $\widetilde{\mathbf{x}}=\left(\widetilde{x}_{1}, \ldots, \widetilde{x}_{n}\right)$. We generate $B$ bootstrap fuzzy random samples $\widetilde{\mathbf{x}}^{*^{1}}, \ldots, \widetilde{\mathbf{x}}^{*^{B}}$ (i.e., each $\widetilde{\mathbf{x}}^{*^{b}}$ is a fuzzy sample of size $n$ randomly drawn with replacement from $\widetilde{\mathbf{x}}$ ) and for each we compute

$$
\chi^{2 *^{b}}=\frac{(n-1) s_{(\widetilde{\mathbf{x}})}^{2 *^{b}}}{s_{(\widetilde{\mathbf{x}})}^{2}}, \quad b=1, \ldots, B
$$

where

$$
s_{(\widetilde{\mathbf{x}})}^{2 *^{b}}=\frac{1}{n-1} \sum_{i=1}^{n} d^{2}\left(\widetilde{x}_{i}^{*^{b}}, \widetilde{\mathbf{x}}^{*}\right), \quad \widetilde{\overline{\mathbf{x}}}^{*}=\frac{1}{n} \sum_{i=1}^{n} \widetilde{x}_{i}^{*^{b}}, \quad s_{(\widetilde{\mathbf{x}})}^{2}=\frac{1}{n-1} \sum_{i=1}^{n} d^{2}\left(\widetilde{x}_{i}, \widetilde{\mathbf{x}}\right),
$$

and $d$ is the Yao-Wu signed distance. The $\gamma$ th percentile of $\chi^{2 *^{b}}$ is estimated by the value $\widehat{t}^{\gamma}$ such that

$$
\frac{\#\left\{\chi^{2 *^{b}} \leq \widehat{t}^{\gamma}\right\}}{B}=\gamma \text {. }
$$

Finally, the crisp bootstrap confidence interval using fuzzy data is

$$
\Pi^{*}=\left[\frac{(n-1) s_{(\widetilde{\mathbf{x}})}^{2}}{\widehat{t}^{1-\gamma}}, \frac{(n-1) s_{(\widetilde{\mathbf{x}})}^{2}}{\widehat{t}^{\gamma}}\right] .
$$

If $B \gamma$ is not an integer, the following procedure can be used. Assuming $\gamma \leq 1 / 2$, let $k=[(B+1) \gamma]$ be the largest integer less or equal $(B+1) \gamma$. Then we define the empirical $\gamma$ and $1-\gamma$ quantiles by the $k$ th and $(B+1-k)$ th largest values of $Z^{*^{b}}$, respectively.

Example 1: Suppose that we have taken a fuzzy random sample of size $n=12$ from a population and that we have observed the triangular fuzzy data of Table 1.

Table 1: Fuzzy random sample of size $n=12$ from a population

\begin{tabular}{|l|l|l|c|r|c|}
\hline & Observation & & Observation & & Observation \\
\hline 1 & $(33,35,36)$ & 5 & $(60,63,66)$ & 9 & $(100,103,105)$ \\
2 & $(80,82,84)$ & 6 & $(70,70,72)$ & 10 & $(54,56,58)$ \\
3 & $(85,87,87)$ & 7 & $(70,73,76)$ & 11 & $(40,40,42)$ \\
4 & $(90,90,90)$ & 8 & $(65,70,73)$ & 12 & $(94,96,99)$ \\
\hline
\end{tabular}

If $B=10000$, the estimates of the $5 \%$ and $95 \%$ percentiles are the 500th and 9500th largest of all $\chi^{2 *^{b}}$ values. The last line of Table 2 shows the percentiles of $\chi^{2 *^{b}}$ for the variance computed using 10000 bootstrap samples.

The bootstrap confidence interval $(\gamma=0.05$ or $90 \%)$ using fuzzy data is

$$
\Pi^{*}=\left(\frac{11 \cdot 444.922}{15.27}, \frac{11 \cdot 444.922}{4.523}\right)=(320.5,1082.1) .
$$

Figure 1 shows the distribution of $\chi^{2 *^{b}}$ computed using 10000 bootstrap samples. 
Table 2: Percentiles of the $\chi_{7}^{2}$ and $\chi_{11}^{2}$ and the bootstrap distribution of $\chi^{2 *^{b}}$

\begin{tabular}{|c|c|c|c|c|c|c|c|c|}
\hline Percentile & 0.005 & 0.01 & 0.025 & 0.05 & 0.95 & 0.975 & 0.99 & 0.995 \\
\hline$\chi_{7}^{2}$ & 0.989 & 1.239 & 1.690 & 2.167 & 14.067 & 16.013 & 18.475 & 20.278 \\
$\chi_{11}^{2}$ & 2.603 & 3.053 & 3.816 & 4.575 & 19.675 & 21.920 & 24.725 & 26.757 \\
Bootstrap & 2.699 & 3.077 & 3.850 & 4.523 & 15.270 & 16.889 & 18.290 & 21.349 \\
\hline
\end{tabular}

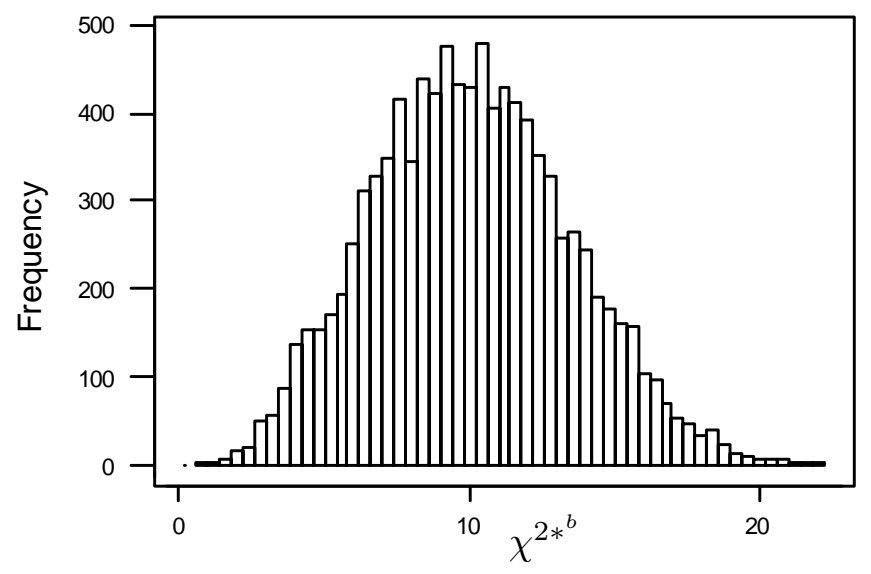

Figure 1: Bootstrap distribution based on $B=10000$ generated $\chi^{2 *^{b}}$ values

\subsection{Fuzzy Confidence Interval}

We generate $B$ bootstrap fuzzy random samples $\widetilde{\mathbf{x}}^{*^{1}}, \ldots, \widetilde{\mathbf{x}}^{*^{B}}$. Then the $\alpha$-cuts of the bootstrap confidence interval using fuzzy data are

$$
\Pi_{\alpha}^{* *}=\left\{\left(\frac{\sum_{i=1}^{n}\left(x_{i}-\overline{\mathbf{x}}\right)^{2}}{\widehat{t}^{1-\gamma}}, \frac{\sum_{i=1}^{n}\left(x_{i}-\overline{\mathbf{x}}\right)^{2}}{\widehat{t}^{\gamma}}\right): x_{i} \in \widetilde{x}_{i \alpha}, i=1, \ldots, n\right\},
$$

whenever its membership function is given by

$$
\mu_{\Pi^{* *}}(y)=\sup _{0 \leq \alpha \leq 1} \alpha I_{\Pi_{\alpha}^{* *}}(y)
$$

Example 2: Consider the sample in Table 1. Now the $\alpha$-cuts of the bootstrap confidence interval $(\gamma=0.05$ or $90 \%)$ using fuzzy data are

$$
\Pi_{\alpha}^{* *}=\left\{\left(\frac{\sum_{i=1}^{12}\left(x_{i}-\overline{\mathbf{x}}\right)^{2}}{15.27}, \frac{\sum_{i=1}^{12}\left(x_{i}-\overline{\mathbf{x}}\right)^{2}}{4.523}\right): x_{i} \in \widetilde{x}_{i \alpha}, i=1, \ldots, n\right\} .
$$

For some $\alpha$ values we get the $\alpha$-cuts as given in Table 3 .

\section{Bootstrap Hypotheses Tests of the Variance}

We now introduce a way to get bootstrap tests for crisp and fuzzy hypotheses based on fuzzy data. 
Table 3: $\alpha$-cuts leading to respective confidence intervals (CIs)

\begin{tabular}{c|cccccc}
\hline$\alpha$ & 0 & 0.1 & 0.2 & 0.3 & 0.4 & 0.5 \\
\hline $\mathrm{CI}$ & $(317.7,1115.8)$ & $(318.1,1112.7)$ & $(318.4,1109.6)$ & $(318.8,1106.6)$ & $(319.2,1103.2)$ & $(319.7,1101.0)$ \\
\hline$\alpha$ & 0.6 & 0.7 & 0.8 & 0.9 & 1 & \\
\hline $\mathrm{CI}$ & $(320.2,1098.4)$ & $(320.7,1095.8)$ & $(321.3,1093.4)$ & $(321.9,1091.1)$ & $(322.5,1088.9)$ & \\
\hline
\end{tabular}

\subsection{Crisp Method and Crisp Hypotheses}

Based on fuzzy observations $\widetilde{\mathbf{x}}=\left(\widetilde{x}_{1}, \ldots, \widetilde{x}_{n}\right)$ we consider an approach to test the following hypotheses:
- $H_{0}: \sigma=\sigma_{0} \quad H_{1}: \sigma \neq \sigma_{0}$,
- $H_{0}: \sigma \geq \sigma_{0} \quad H_{1}: \sigma<\sigma_{0}$,
- $H_{0}: \sigma \leq \sigma_{0} \quad H_{1}: \sigma>\sigma_{0}$.

Decision rule: We know that $\Pi^{*}$ is a crisp confidence interval, thus

- if $\sigma_{0}^{2} \in \Pi^{*}$, we accept $H_{0}$,

- if $\sigma_{0}^{2} \notin \Pi^{*}$, we reject $H_{0}$.

Example 3: Consider the sample in Table 1. Suppose we are interested in a bootstrap test for the hypotheses

$$
H_{0}: \sigma=27 \quad H_{1}: \sigma \neq 27 .
$$

Since we have $729 \in[320.5,1082.1]$, we accept $H_{0}$.

\subsection{Fuzzy Method and Crisp Hypotheses}

According to the hypotheses in Subsection 4.1, we consider the problem in the following way:

\section{Decision rule:}

- if $\mu_{\Pi^{* *}}\left(\sigma_{0}^{2}\right)<1 / 2$, we reject $H_{0}$ with degree of rejection (DoR) $1-\mu_{\Pi^{* *}}\left(\sigma_{0}^{2}\right)$,

- if $\mu_{\Pi^{* *}}\left(\sigma_{0}^{2}\right)>1 / 2$, we accept $H_{0}$ with degree of acceptance (DoA) $\mu_{\Pi^{* *}}\left(\sigma_{0}^{2}\right)$,

- if $\mu_{\Pi^{* *}}\left(\sigma_{0}^{2}\right)=1 / 2$, we accept $H_{0}$ or $H_{1}$.

Example 4: Consider the sample in Table 1. Suppose we are interested in a bootstrap test for the hypotheses

$$
H_{0}: \sigma=17.9 \quad H_{1}: \sigma \neq 17.9 .
$$

We use the ability of the package Maple 7 and verify that $\mu_{\Pi^{* *}}(17.9)=0.684$ and that $1-\mu_{\Pi^{*}}(17.9)=0.316$. Thus, we accept $H_{0}$ with DoA 0.684 . 


\subsection{Fuzzy Method and Fuzzy Hypotheses}

We define some models as fuzzy sets of real numbers for modelling the extended versions of the simple, the one-, and the two-sided ordinary (crisp) hypotheses to the fuzzy ones.

Testing statistical hypotheses is a main topic in statistical inference. Typically, a statistical hypothesis is an assertion about the probability distribution of random variables. Traditionally, all statisticians assume that the hypothesis (for which we want to provide a test) are well-defined. Sometimes, this limitation force the statistician to make decision procedures in an unrealistic manner. This is because in realistic problems, we may come across with non-precise (fuzzy) hypotheses. For example, suppose that $\theta$ is the proportion of a population with a disease. We take a random sample and study this sample in order to have some idea about $\theta$. In crisp hypotheses testing one uses hypotheses of the form $H_{0}: \theta=0.2$ versus $H_{1}: \theta \neq 0.2$ or $H_{0}: \theta \leq 0.2$ versus $H_{0}: \theta>0.2$, and so on. However, we sometimes like to test more realistic hypotheses. In this example, more realistic expressions about $\theta$ would be considered as small, very small, large, approximately 0.2 , and so on. Therefore, a more realistic formulation of the hypotheses might be $H_{0}: \theta$ is small versus $H_{1}: \theta$ is not small. We call such expressions fuzzy hypotheses.

Definition 4: Let $\theta_{0}$ be a real known number. A hypothesis of the form

- " $H: \theta$ is approximately $\theta_{0}$ " is called a fuzzy simple hypothesis.

- " $H: \theta$ is not approximately $\theta_{0}$ " is called a fuzzy two-sided hypothesis.

- " $H$ : $\theta$ is essentially smaller than $\theta_{0}$ " is called a fuzzy left one-sided hypothesis.

- " $H$ : $\theta$ is essentially larger than $\theta_{0}$ " is called a fuzzy right one-sided hypothesis.

We denote the above definitions by

$H_{0}: \theta$ is approx. $\theta_{0}$ vs. $H_{1}: \theta$ is not approx. $\theta_{0}$, or $H_{0}: \theta$ is $\widetilde{H}_{0}$ vs. $H_{1}: \theta$ is $\widetilde{H}_{1}$, $H_{0}: \theta$ is approx. $\theta_{0}$ vs. $H_{1}: \theta$ is certainly larger than $\theta_{0}$, or $H_{0}: \theta$ is $\widetilde{H}_{0 L}$ vs. $H_{1}: \theta$ is $\widetilde{H}_{1}$, $H_{0}: \theta$ is approx. $\theta_{0}$ vs. $H_{1}: \theta$ is certainly smaller than $\theta_{0}$, or $H_{0}: \theta$ is $\widetilde{H}_{0 R}$ vs. $H_{1}: \theta$ is $\widetilde{H}_{1}$.

These fuzzy hypotheses are shown in Figures 2 to 4.

Consider the problem of testing the fuzzy hypotheses $\widetilde{H}_{0}$ versus $\widetilde{H}_{1}$ based on a fuzzy random sample. $\widetilde{H}_{0 L}$ versus $\widetilde{H}_{1}$ and $\widetilde{H}_{0 R}$ versus $\widetilde{H}_{1}$ are similar to $\widetilde{H}_{0}$ versus $\widetilde{H}_{1}$.

Assumptions: Let

- $C_{T}$ be the total area under $\widetilde{H}_{0}$,

- $C_{A}$ be the area of the intersection between $\widetilde{H}_{0}$ and $\mu_{\Pi^{* *}}$,

- $C_{R}$ be the area of the intersection between $\widetilde{H}_{0}$ and $1-\mu_{\Pi^{* *}}$.

We know that $\widetilde{H}_{0}$ and $\Pi^{* *}$ are canonical fuzzy numbers, thus the areas $C_{T}, C_{A}$ and $C_{R}$ are finite.

\section{Decision rule:}

- if $C_{T}=C_{A}$ and $C_{R}=\emptyset$, then we accept $H_{0}$.

- if $C_{T}=C_{R}$ and $C_{A}=\emptyset$, then we reject $H_{0}$.

- if $C_{A} / C_{R}>1$, then we accept $H_{0}$ with DoA $C_{A} / C_{T}$.

- if $C_{A} / C_{R}<1$, then we reject $H_{0}$ with DoR $C_{R} / C_{T}$.

- if $C_{A} / C_{R}=1$, then we accept $H_{0}$ and $H_{1}$. 


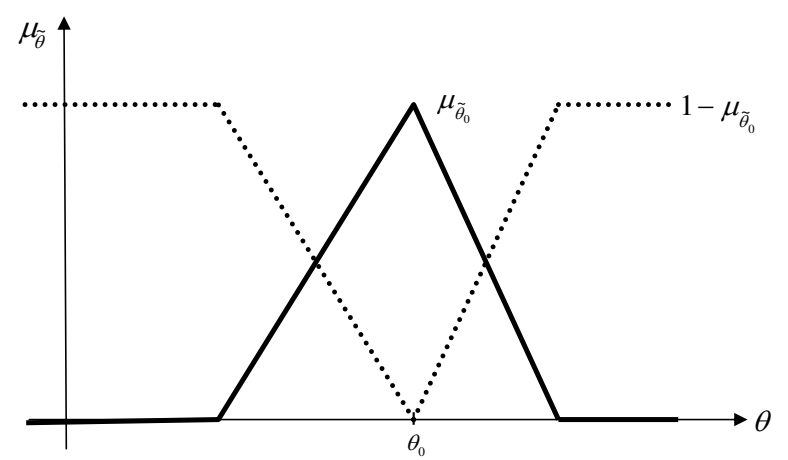

Figure 2: Fuzzy hypothesis $\widetilde{H}_{0}$ versus $\widetilde{H}_{1}$

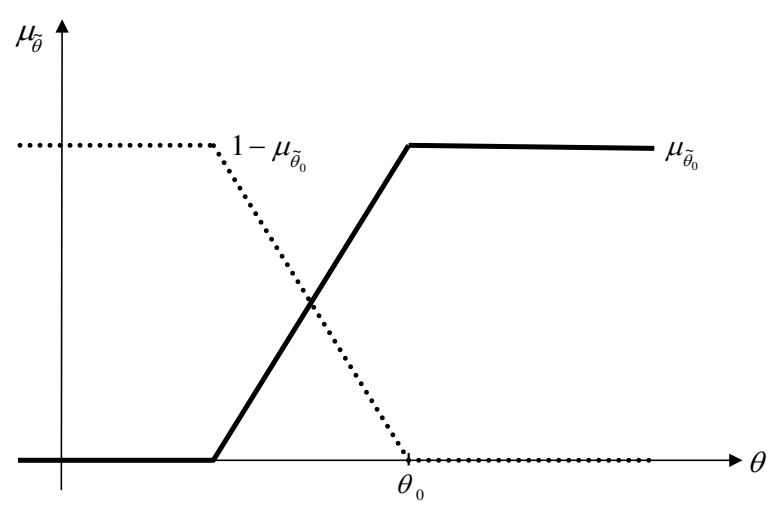

Figure 3: Fuzzy hypothesis $\widetilde{H}_{0 L}$ versus $\widetilde{H}_{1}$

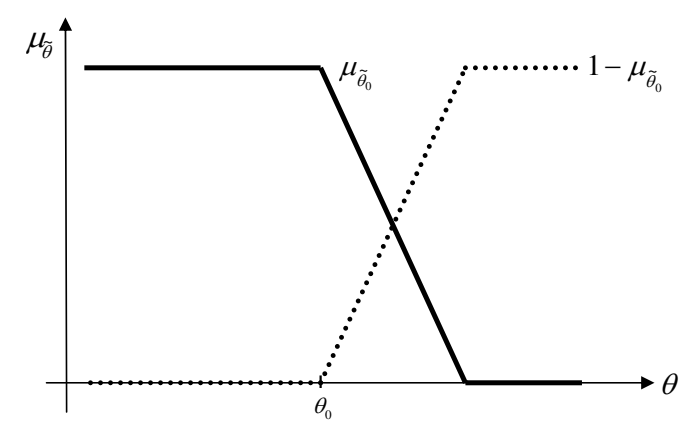

Figure 4: Fuzzy hypothesis $\widetilde{H}_{0 R}$ versus $\widetilde{H}_{1}$

If $C_{T}=C_{A}$ and $C_{R}=\emptyset$, then the fuzzy number $\widetilde{\theta}_{0}$ lies in the fuzzy confidence interval $\Pi^{* *}$ and the null hypothesis $\widetilde{H}_{0}$ is certainly accepted with DoA $C_{A} / C_{T}=1$. On the other hand, if $C_{A}$ decreases then the value $C_{R} / C_{T}$ increases and we certainly reject $\widetilde{H}_{0}$ with DoR $C_{R} / C_{T}=1$ when $C_{T}=C_{R}$ and $C_{A}=\emptyset$. In other words, the value $\widetilde{\theta}_{0}$ lies in the fuzzy confidence interval $1-\Pi^{* *}$.

Taking greater membership functions 0.7 or 0.8 for $\widetilde{\theta}_{0}$ and $\Pi^{* *}$ we could reach more accurate values of $C_{T}, C_{A}$, and $C_{R}$. In summary, the above procedure is an applicable tool in fuzzy statistical inferential schemes. In the end of paper, we exhibit a decision making method which for $\alpha=1$ is the same as under classical procedures.

Example 5: Consider the sample in Table 1. Now suppose that we want to test the fuzzy hypotheses

$$
H_{0}: \sigma \text { is }(25,30,55) \quad H_{1}: \sigma \text { is not }(25,30,35) \text {. }
$$


Here, $H_{0}$ suggests that $\sigma$ is approximatively 30, and $H_{1}$ suggests that $\sigma$ is away from 30 . Hence, based on the ability of Maple 7 we have $C_{A} / C_{R}=1.97>1$. Thus, we accept $H_{0}$ with DoA $C_{A} / C_{T}=0.865$. Figure 5 shows the distribution of the membership function $\mu_{\Pi^{* *}}$ and fuzzy hypotheses $\widetilde{H}_{0}$ versus $\widetilde{H}_{1}$. Figure 6 shows essentially the same but the plot is based on a larger membership function of 0.42 . Hence, we have $C_{A} / C_{R}=13>1$. Thus, we accept $H_{0}$ with DoA $C_{A} / C_{T}=0.914$.

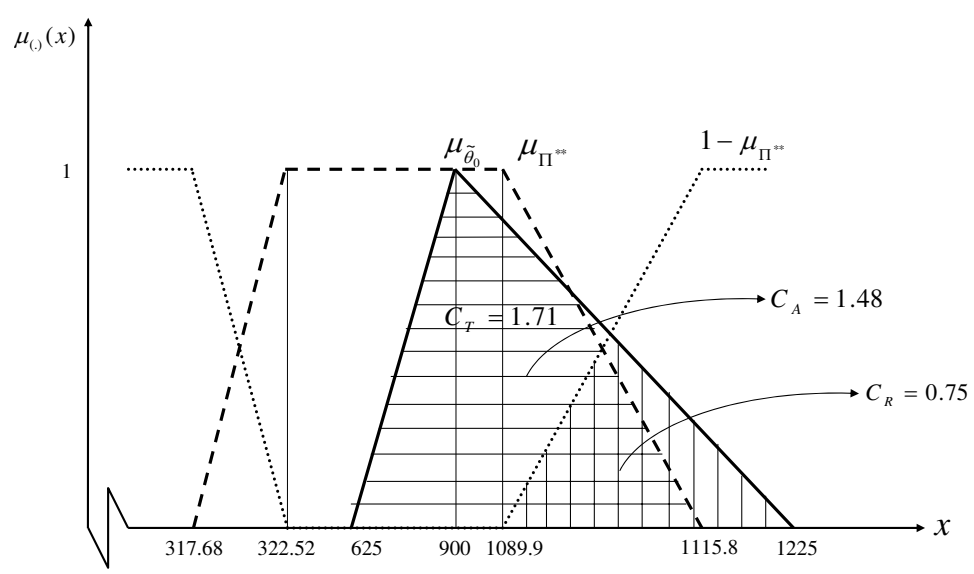

Figure 5: Membership function $\mu_{\Pi^{* *}}$ and fuzzy hypotheses $H_{0}$ versus $H_{1}$

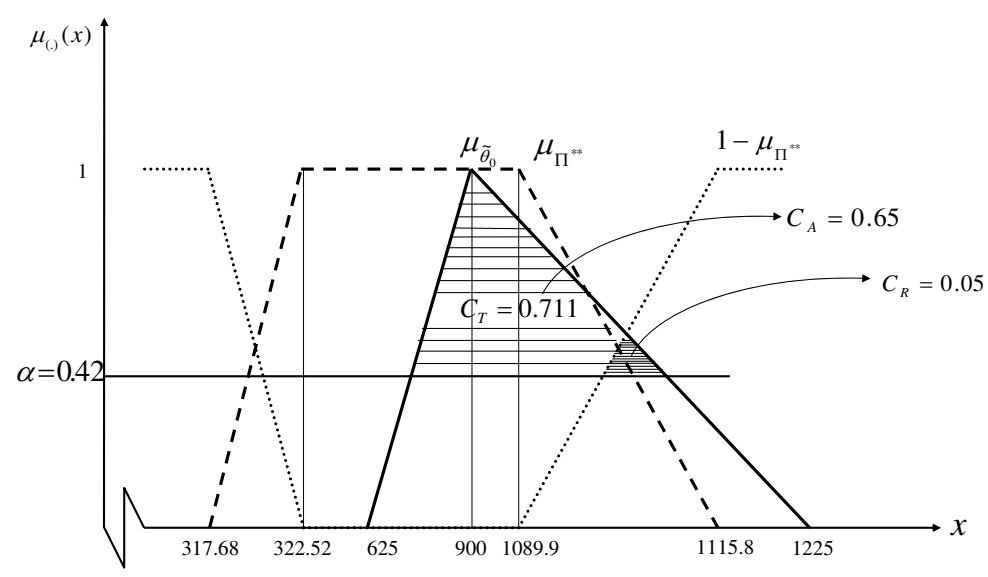

Figure 6: Membership function $\mu_{\Pi^{* *}}$ and fuzzy hypotheses $H_{0}$ versus $H_{1}$ with $\alpha=0.42$

\section{Conclusions}

The new approach for bootstrap statistical inference for the variance based on fuzzy data has the following issues:

1. It is established upon the notion of crisp and fuzzy confidence intervals (note that, in classical testing hypotheses, there is a relationship between interval estimation and testing hypothesis).

2. By introducing the concepts of DoA and DoR, it enables us to test fuzzy hypotheses in a rather natural way. 
This procedure is based on the relationship between interval estimation and hypothesis tests in fuzzy environments. Extension of the proposed method to test the variance, correlation, and parameters in linear regression models is a potential area for future work.

\section{References}

Buckley, J. J. (2005). Fuzzy Probabilities: New Approach and Applications. Berlin, Heidelberg: Springer.

Buckley, J. J. (2006). Fuzzy Probability and Statistics. Berlin, Heidelberg: Springer.

Cheng, C. (1998). A new approach for ranking fuzzy numbers by distance method. Fuzzy Sets and Systems, 95, 307-317.

Filzmoser, P., and Viertl, R. (2004). Testing hypotheses with fuzzy data: the fuzzy pvalue. Metrika, 59, 21-29.

Gonzalez-Rodriguez, G., Montenegro, M., Colubi, A., and Gil, M. A. (2006). Bootstrap techniques and fuzzy random variables: Synergy in hypothesis testing with fuzzy data. Fuzzy Sets and Systems, 157, 2608-2613.

Körner, R. (2000). An asymptotic $\alpha$-cut for the expectation of random fuzzy variables. Journal of Statistical Planning and Inference, 83, 331-346.

Modarres, M., and Sadi-Nezhad, S. (2001). Ranking fuzzy numbers by preference ratio. Fuzzy Sets and Systems, 118, 429-436.

Montenegro, M., Colubi, A., Casals, M. R., and Gil, M. A. (2004). Asymptotic and bootstrap techniques for testing the expected value of a fuzzy random variable. Metrika, 59, 31-49.

Nojavan, M., and Ghazanfari, M. (2006). A fuzzy ranking method by desirability index. Journal of Intelligent and Fuzzy Systems, 17, 27-34.

Taheri, S. M., and Arefi, M. (2008). Testing fuzzy hypotheses based on fuzzy statistics. Soft Computing, 13, 617-625.

Thompson, E. A., and Geyer, C. J. (2007). Fuzzy p-values in latent variable problems. Biometrika, 94, 49-60.

Torabi, H., Behboodian, J., and Taheri, S. M. (2006). Neyman-Pearson lemma for fuzzy hypotheses testing withy vague data. Metrika, 64, 289-304.

Viertl, R. (2006). Univariate statistical analysis with fuzzy data. Computational Statistics and Data Analysis, 51, 133-147.

Yao, J. S., and Wu, K. (2000). Ranking fuzzy numbers based on decomposition principle and signed distance. Fuzzy Sets and Systems, 11, 275-288.

Authors' Addresses:

Mohammad Ghasem Akbari

Abdolhamid Rezaei

Department of Statistics

Department of Statistics

Faculty of Sciences

School of Mathematical Sciences

University of Birjand

Ferdowsi University of Mashhad

South Khorasan, Iran

Mashhad, Iran

E-mail: g_z_akbari@yahoo.com

E-mail: rezaei494@gmail.com 